2014

Local Covariance Estimation Using Costationarity

\author{
Cardinali, Alessandro
}

http://hdl.handle.net/10026.1/13473

10.1007/978-1-4939-0569-0_6

Springer Proceedings in Mathematics and Statistics

Springer New York

All content in PEARL is protected by copyright law. Author manuscripts are made available in accordance with publisher policies. Please cite only the published version using the details provided on the item record or document. In the absence of an open licence (e.g. Creative Commons), permissions for further reuse of content should be sought from the publisher or author. 


\title{
Local Covariance Estimation using Costationarity
}

\author{
Alessandro Cardinali
}

\begin{abstract}
In this paper we propose a novel estimator for the time-varying covariance of locally stationary time series. This new approach is based upon costationary combinations of such time series, that is, time-varying deterministic combinations returning stationary processes. We show with a simulation example that the new estimator has smaller variance than the available competitors which are exclusively based on the evolutionary cross-periodogram, and can therefore be appealing in a large number of applications.
\end{abstract}

\section{Introduction}

Loosely speaking, a stationary time series is one whose statistical properties remain constant over time. A locally stationary (LS) time series is one whose statistical properties can change slowly over time. As a consequence, such a series can appear stationary when examined close up, but appear non-stationary when examined on a larger scale. Priestley (1983) and Priestley (1988) provide a comprehensive review of locally stationary processes and their history, Nason and von Sachs (1999) provides a more recent review. The methods described in this article can be applied to locally stationary time series that are a triangular stochastic arrays defined in the rescaled time $t / T$, where $T$ represents the sample size.

Based on this setup, Dahlhaus (1997) proposed locally stationary Fourier (LSF) processes whose underlying pseudo-spectral structure is defined in terms of Fourier basis. The locally stationary wavelet (LSW) model due to Nason et al. (2000), instead, decomposes the local structure of the process among different scales through a set of non-decimated wavelets used as basis functions. In the following, we will consider the latter family of processes defined as

Alessandro Cardinali

University of Bristol, e-mail: a.cardinali@bristol.ac.uk 


$$
X_{t ; T}=\sum_{j=1}^{\infty} \sum_{k=-\infty}^{\infty} W_{j}\left(\frac{k}{T}\right) \psi_{j}(t-k) \varepsilon_{j, k}
$$

where $\left\{\psi_{j}(t-k)\right\}_{j}$ is a family of discrete non-decimated wavelet filters whose local support includes the neighborhood of $t$, and is spanned by the index $k$. The parameter $j$ is integer valued and represents the scale of the corresponding wavelet. The function $W_{j}(k / T)$ is a time localized amplitude of bounded variation, referring to diadic scales indexed by $j$. Finally $\varepsilon_{j, k}$ is a sequence of doubly indexed i.i.d. standardized random variables. This setup allows the definition of a time-varying generalization of the classical spectra having a well defined limit in the rescaled time $z \in(0,1)$ defined as

$$
S_{j}(z)=\lim _{T \rightarrow \infty}\left|W_{j}\left(\frac{[z T]}{T}\right)\right|^{2},
$$

where we have set $k=[z T]$, and $[x]$ is the integer part of $x$. This multiscale LS framework has proven to be useful to estimate the time-varying association between non-stationary time series. The estimation of the local covariance is relevant in a wide range of disciplines such as climatology, neuroscience and economics, where the underlying phenomena are inherently characterized by regime changes that cannot be appropriately taken into account by classical stationary models.

Ombao and Van Bellegem (2006) and Sanderson et al. (2010) propose methodologies based, respectively, on the cross-spectra of LSF and LSW models. In this paper we propose an alternative methodology to estimate the local covariance function, which is based on the existence of time-varying linear combinations of LS processes which are (co)stationary, see Cardinali and Nason (2010). The contribution of this paper is therefore twofold. We first propose the new estimation methodology, and then show this to be statistically efficient in comparison to the method proposed in Sanderson et al. (2010). We illustrate a theoretical example and then validate the comparison by means of simulations.

The article is structured as follows. Section 2 reviews the second order properties of LS time series models and briefly describes the concept of costationarity. Section 3 introduce our new covariance estimator, and illustrate its relative efficiency over the classical estimators using a theoretical and simulation example.

\section{Local Covariance and Costationarity}

When processes are not stationary in the wide sense, covariance operators may have complicated time-varying properties. With non-stationarity their estimation is typically difficult, since a canonical spectral structure does not exist. Meyer (1993) showed that although we are not able to find the bases which diagonalize complicated integral operators in general, it is nevertheless possible to find well structured

bases which compress them. This means that (local) covariance operators can be well represented by sparse matrices with respect to such bases. For $n=1,2, \ldots, N$, 
the locally stationary behavior of LS processes $X_{t ; T}^{(n)}$ is characterized by a local autocovariance function

$$
\gamma_{T}(t, \tau)=\gamma_{n, n ; T}(t, \tau)=\operatorname{Cov}\left(X_{t ; T}^{(n)}, X_{t+\tau ; T}^{(n)}\right) .
$$

Using the approximation derived in Sanderson et al. (2010) this is representable as

$$
\gamma_{T}(t, \tau)=\sum_{j=1}^{\log _{2} T} S_{j}\left(\frac{t}{T}\right) \Psi_{j}(\tau)+\mathscr{O}\left(T^{-1}\right)
$$

where $S_{j}(t / T)=S_{j}(t / T)^{(n)}$ is the local spectra for $X_{t ; T}^{(n)}$, and $\Psi(\tau)=\sum_{t} \psi_{j}(t) \psi_{j}(t+$ $\tau)$ is the autocorrelation wavelet, see Nason et al. (2000) for details. Similarly, using another approximation derived in Sanderson et al. (2010), for $n, m=1,2, \ldots, N$, the local cross-covariance between two locally stationary processes $X_{t ; T}^{(n)}$ and $X_{t ; T}^{(m)}$ can be defined as

$$
\gamma_{n, m ; T}(t, \tau)=\sum_{j=1}^{\log _{2} T} S_{j}^{(n, m)}\left(\frac{t}{T}\right) \Psi_{j}(\tau)+\mathscr{O}\left(T^{-1}\right),
$$

where $S_{j}^{(n, m)}(t / T)$ is he local cross-spectra defined as

$$
S_{j}^{(n, m)}(t / T)=W_{j}^{(n)}\left(\frac{t}{T}\right) W_{j}^{(m)}\left(\frac{t}{T}\right),
$$

where $W_{j}^{(n)}(t / T)$ and $W_{j}^{(m)}(t / T)$ are the local amplitude functions for the processes $X_{t ; T}^{(n)}$ and $X_{t ; T}^{(m)}$ respectively. Note that these functions, along with the local spectra and cross-spectra, are defined in the rescaled time, and their limits for $T \rightarrow \infty$ are well defined as $\gamma(z, \tau), \gamma_{n, m}(z, \tau), S_{j}(z)$ and $S_{j}^{(n, m)}(z)$ respectively, for $z \in(0,1)$. Moreover, note also that for univariate (globally) stationary time series the spectra $S_{j}(t / T)$ is time invariant, i.e. $S_{j}(t / T)=S_{j} \forall j$, which also implies for the same process to have a Toeplitz covariance operator $\gamma(t, \tau)=\gamma(\tau)$. Finally, the local covariance between two LS processes can be obtained as a particular case of equation (4), when we set $\tau=0$, and is therefore defined as

$$
\gamma_{n, m ; T}(t, 0)=\sum_{j=1}^{\log _{2} T} S_{j}^{(n, m)}\left(\frac{t}{T}\right)+\mathscr{O}\left(T^{-1}\right) .
$$

\subsection{Costationarity}

We give a multivariate extension for the definition of costationary processes originally proposed in Cardinali and Nason (2010). However, we now concentrate on constant piecewise solution vectors. 
Definition 1 Let $\mathbf{X}_{t ; T}=\left(X_{t ; T}^{(1)}, \ldots, X_{t ; T}^{(N)}\right)$ be a vector time series with local autocovariances and cross-covariances satisfying equations (3) and (4). Moreover assume

$$
\sup _{t}\left|\operatorname{cor}\left(X_{t ; T}^{(n)}, X_{t ; T}^{(m)}\right)\right|<1,
$$

and for $n, m=1,2, \ldots, N$. We call $Z_{t}^{(i)}$ costationary process if there exists a set of bounded piecewise constant functions $\alpha_{t}^{(n, i)}$ for $t=1, \ldots, T, n=1, \ldots, N$ and $i=$ $1,2, \ldots$, I such that

$$
Z_{t}^{(i)}=\sum_{n=1}^{N} \alpha_{t}^{(n, i)} X_{t ; T}^{(n)}
$$

is a covariance stationary process.

Costationary solutions are, in general, multiple. This multiplicity is represented here through the index $i$. Using a vector notation we can also represent the set of costationary solutions as a time-varying linear system

Definition 2 Let $\mathbf{Z}_{t}=\left(Z_{t}^{(1)}, \ldots, Z_{t}^{(I)}\right)$ and $\mathbf{X}_{t ; T}$ as in Definition 1. We define the costationary system as

$$
\mathbf{Z}_{t}=\mathbf{A}_{t} \mathbf{X}_{t ; T}
$$

where, for each time point $t, \mathbf{A}_{t}=\left(\alpha_{i, n ; t}\right)_{i, n}$ is a $(I \times N)$ dimensional matrix of costationary vector entries for time $t$.

The piecewise constant functions $\alpha_{t}^{(n, i)}$ are supposed to be measurable on a disjoint sequence of half-opened diadic intervals. In this paper intervals of dyadic length have been considered for computational convenience, however, in principle the theory we present will apply to intervals of arbitrary length. For a discussion on segmentation issues and regularity conditions concerning costationary solutions $\alpha_{t}^{(n, i)}$ we refer again the interested reader to Cardinali and Nason (2010). For an arbitrary time-varying LS combination, the local variance can be represented as

$$
\sigma_{Z_{i}}^{2}(t)=\sum_{n, m} \alpha_{t}^{(n, i)} \alpha_{t}^{(m, i)} \gamma_{n, m ; T}(t, 0),
$$

for $n, m=1, \ldots, N$, and is typically a time-varying quantity. However, when considering costationary combinations $Z^{(i)}(t)$, the quantity defined in equation (7) becomes time-invariant, so we can simply refer to it as $\sigma_{Z_{i}}^{2}$.

\section{A Costationary Estimator for Local Covariances}

A direct estimator for the time-varying covariance of locally stationary processes can be obtained by plugging a, possibly unbiased and consistent, cross-periodogram into equation (6). The aim of this section is to introduce an alternative estimator for the local covariance which makes use of costationary solutions. We will use integers 
$n, m, l, h=1,2, \ldots, N$ to identify (pairs of) LS processes. By imposing costationarity the quantity defined in equation (7) can be estimated using the plug-in estimator

$$
\hat{\sigma}_{Z_{i}}^{2}=\sum_{n, m} \hat{\alpha}_{t}^{(n, i)} \hat{\alpha}_{t}^{(m, i)} \hat{\gamma}_{n, m ; T}(t, 0)
$$

where $\hat{\gamma}_{n, m ; T}(t, 0)$ can be any asymptotically unbiased and consistent estimator for $\gamma_{n, m ; T}(t, 0)$ and $\hat{\alpha}_{t}^{(n, i)}$ can be determined using the costat algorithm described in Cardinali and Nason (2010). In practice we will obtain $\hat{\sigma}_{Z_{i}}^{2}$ using the sample variance estimator of costationary processes $Z_{t}^{(i)}$. However, from equation (8), we can obtain an alternative local covariance estimator as

$$
\hat{\gamma}_{l, h ; T}^{(i)}(t, 0)=\frac{\hat{\sigma}_{Z_{i}}^{2}-\sum_{n \neq l, m \neq h} \hat{\alpha}_{t}^{(n, i)} \hat{\alpha}_{t}^{(m, i)} \hat{\gamma}_{n, m ; T}(t, 0)}{\hat{\alpha}_{t}^{(l, i)} \hat{\alpha}_{t}^{(h, i)}} .
$$

We are particularly interested in assessing any gain in efficiency possibly due to averaging these single-solution estimators over multiple solutions. We will therefore consider the estimator

$$
\hat{\gamma}_{l, h ; T}^{*}(t, 0)=\frac{1}{I} \sum_{i=1}^{I} \hat{\gamma}_{l, h ; T}^{(i)}(t, 0)
$$

The following section illustrates the efficiency of this estimator with a theoretical and simulation example.

\subsection{Theoretical example and simulations}

For the illustrative purposes of this example we will only consider time-invariant costationary systems, i.e. costationary systems with time-invariant costationary vectors. These systems can be defined, as a particular case of the general form given in Definition 2, as

$$
\mathbf{Z}_{t}=\mathbf{A} \mathbf{X}_{t ; T},
$$

We consider two uncorrelated LS Gaussian processes $X_{t ; T}^{(1)}$ and $X_{t ; T}^{(2)}$, respectively having local spectra

$$
S_{j}^{(1)}(t / T)= \begin{cases}2 / 3, & \text { if } j=j^{*} \text { and } t \leq T / 2 \\ 1 / 3, & \text { if } j=j^{*} \text { and } t>T / 2 \\ 0, & \text { otherwise. }\end{cases}
$$

and 


$$
S_{j}^{(2)}(t / T)= \begin{cases}1 / 3, & \text { if } j=j^{*} \text { and } t \leq T / 2 \\ 2 / 3, & \text { if } j=j^{*} \text { and } t>T / 2 \\ 0, & \text { otherwise. }\end{cases}
$$

Then, for $i=1,2,3,4$, and time-invariant costationary vectors

$$
\begin{aligned}
& \alpha^{(1)}=(1,1) \\
& \alpha^{(2)}=(1,-1) \\
& \alpha^{(3)}=(-1,1) \\
& \alpha^{(4)}=(-1,-1)
\end{aligned}
$$

the linear combinations $Z_{t}^{(i)}$ are White-Noise processes with variance $\sigma_{Z_{i}}^{2}=1$. This is an example of multiple costationary solution vectors which are also timeinvariant. Other costationary vectors can be found by multiplying $\alpha^{(i)}$ by some real scalar. By applying the singular value decomposition $\mathbf{A}=\mathbf{U} \mathbf{D} \mathbf{V}$ we obtain

$$
\begin{aligned}
\mathbf{A} & =\left(\begin{array}{rr}
1 & 1 \\
1 & -1 \\
-1 & 1 \\
-1 & -1
\end{array}\right) \\
& =\left(\begin{array}{rr}
-1 / 2 & 1 / 2 \\
-1 / 2 & -1 / 2 \\
1 / 2 & 1 / 2 \\
1 / 2 & -1 / 2
\end{array}\right)\left(\begin{array}{ll}
2 & 0 \\
0 & 2
\end{array}\right)\left(\begin{array}{rr}
-1 & 0 \\
0 & 1
\end{array}\right)
\end{aligned}
$$

In this case we therefore have $\operatorname{rank}(\mathbf{A})=2$ and we expect the estimator $\hat{\gamma}_{l, h ; T}^{*}(t, 0)$ to be more efficient that the direct estimator proposed in Sanderson et al. (2010). This is because the degree of efficiency of $\hat{\gamma}_{l, h ; T}^{*}(t, 0)$, is obtained by exploiting the number of non-perfectly correlated LS time series $X_{t ; T}^{(n)}$, which are used to produce synthetic linearly independent data.

Remark 1 Note that, even in the general time-varying case of Definition 1 (i.e. when $\mathbf{A}_{t}$ depends upon time), conditions therein are sufficient to ensure that $\operatorname{rank}\left(\mathbf{A}_{t}\right)=$ $N$, for all $t=1, \ldots, T$, provided that $I \geq N$. The greater is the number of non-perfectly correlated time series returning costationary solutions, the greater is the degree of efficiency of the new estimator.

We use this simple theoretical example to conduct a simulation experiment investigating the relative efficiency of our costationary estimator for the local covariance function when compared with the classical estimator exclusively based on the local cross-spectra, that is, the plug-in estimator of the quantity defined in equation (6). We simulate pairs of uncorrelated multiscale LS Gaussian processes having local spectra as defined in equations (12) and (13). 
In this experiment we use a (real-valued) wavelet model representation. We choose $j^{*}=4$ and simulate $30 \times 29 / 2=435$ pairs of uncorrelated processes of increasing length. For each length, and for each pair, we first estimate the local covariance by using the classical local estimator. We then repeat the estimation by using the statistics defined in equation (10). We use simulations to compute the timeaveraged Montecarlo variance of each estimator and to estimate the time-averaged relative efficiency ratio. The analysis is conducted on samples of increasing size $T=128,256,512,1024,2048$. The results of this experiment are displayed in Table 1 . The results of our simulations show that the new estimator is substantially

\begin{tabular}{|l|ccccc|}
\hline \hline Estimates & $\mathrm{T}=128$ & $\mathrm{~T}=256$ & $\mathrm{~T}=512$ & $\mathrm{~T}=1024$ & $\mathrm{~T}=2048$ \\
\hline $\operatorname{var}[\hat{\gamma}(t, 0)]$ & 0.0092 & 0.0101 & 0.0103 & 0.0112 & 0.0134 \\
$\operatorname{var}\left[\hat{\gamma}^{*}(t, 0)\right]$ & 0.0029 & 0.0028 & 0.0029 & 0.0030 & 0.0032 \\
$\operatorname{eff}\left[\hat{\gamma}(t, 0) / \hat{\gamma}^{*}(t, 0)\right]$ & 3.1710 & 3.5970 & 3.5579 & 3.7321 & 4.1934 \\
\hline \hline
\end{tabular}

Table 1 Time-averaged Montecarlo variances and relative efficiency ratios for classical and costationary local covariance estimators

more efficient than the estimator exclusively based upon the cross-periodogram. Interestingly, the gain in efficiency is substantial even for very moderate sample sizes. Moreover, increasing efficiency can be achieved by considering a larger number of time series in costationary combinations. Future work will consider a full theoretical investigation of this approach as well as some applications to economics and financial data.

Acknowledgements I am grateful to Guy Nason for reading a preliminary version of the manuscript and for providing useful comments. All errors are my own responsibility.

\section{References}

Brillinger, D. (2001). Time Series: Data Analysis and Theory. SIAM, Philadelphia. Cardinali, A. and Nason, G. P. (2010). Costationarity of Locally Stationary Time Series. J. of Time Series Econometrics, 2(2):1-19.

Cardinali, A. and Nason, G. P. (2012). Costationarity of Locally Stationary Time Series using Costat. Preprint.

Dahlhaus, R. (1997). Fitting time series models to nonstationary processes. Ann. Statist., 25:1-37.

Fuller, W. (1996). Introduction to Statistical Time Series. Wiley, New York.

Granger, C.W, (2008). Non-linear models, where do we go next: time-varying parameter models? Studies in Nonlinear Dynamics \& Econometrics, 12(3):1-9.

Meyer, Y. (1993). Wavelets and operators. Proceedings of Symposia in Applied Mathematics,47, 35-57. 
Nason, G. and von Sachs, R. (1999). Wavelets in time series analysis. Phil. Trans. R. Soc. Lond. A, 357:2511-2526.

Nason, G., von Sachs, R., and Kroisandt, G. (2000). Wavelet processes and adaptive estimation of the evolutionary wavelet spectrum. J. R. Statist. Soc. B, 62:271-292.

Nason, G. P. (2008) Wavelet Methods in Statistics with R. Springer, New York.

Ombao, H. and Van Bellegem, S. (2006). Coherence analysis of nonstationary time series: a linear filtering point of view. Discussion Paper DP0618.

Priestley, M. (1983). Spectral Analysis and Time Series. Academic Press.

Priestley, M. (1988). Non-linear and Non-stationary Time Series Analysis. Academic Press.

J. Sanderson, J., Fryzlewicz, P. and Jones, M. (2010). Estimating linear dependence between nonstationary time series using the locally stationary wavelet model. Biometrika, 97:435-446. 